\title{
The Autonomous Micro Robot "Alice": a platform for scientific and commercial applications.
}

\author{
G. Caprari, P. Balmer, R. Piguet, R. Siegwart \\ Swiss Federal Institute of Technology Lausanne (EPFL) \\ Autonomous System Lab \\ EPFL, DMT-ISR, CH-1015 Lausanne \\ E-mail: Gilles.Caprari@epfl.ch \\ http://dmtwww.epfl.ch/isr/asl
}

\begin{abstract}
:
Over the last years various mobile micro robots have been developed at different labs for research issues. Most of them have remarkable features but are handmade prototypes not suitable for large-scale production.

This paper presents a micro robot design where major efforts have been undertaken in order to reduce costs and facilitate serial production, while improving the performances of the robot. The newly designed robot consists of only three major parts, the flexible print with the electronics and the two motors directly mounted on it, the plastic frame on which the print is attached by four screws and the battery. Thanks to its very low costs, the micro robot is of high interest for multiple robot applications, for investigation of collective behavior and for the toy market.

Our robot Alice size about $2 \times 2 \times 2 \mathrm{~cm}$, has a ultra low power consumption allowing autonomy up to 10 hours, and has a modular concept for programming and hardware extension. This makes it very useful for research and future developments. We extended the robot abilities with 4 distance sensors, an infrared communication module and a radio communication module. By only modifying the software, many additional functions are available. Some of them are presented such as obstacle avoidance, wall following, remote inspection and guidance, communication between robots and multi-robot exploration.
\end{abstract}

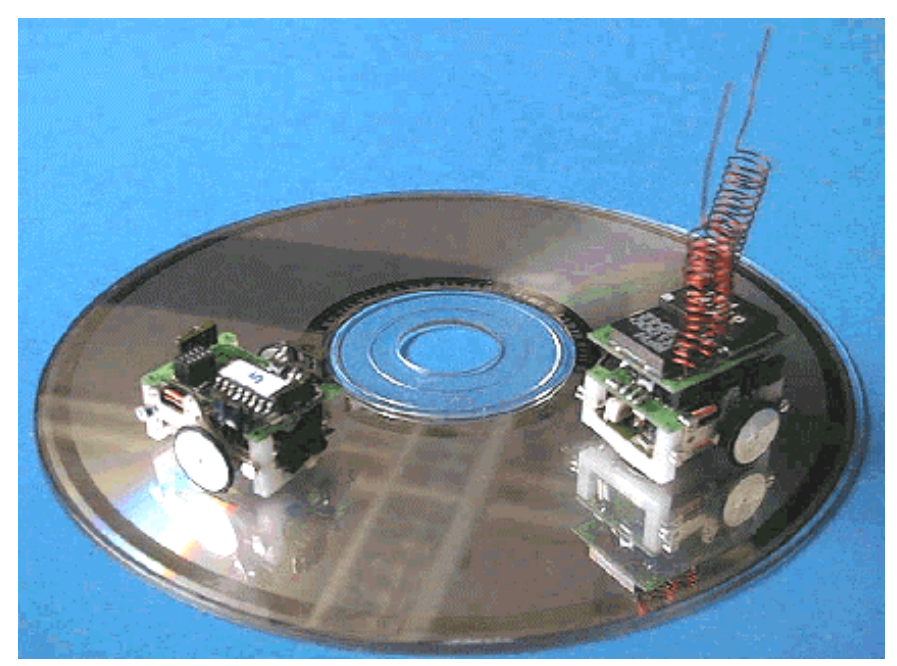

Figure 1. Two Alices on a CD-ROM

\section{Introduction}

In the last years many new highly integrated electronic devices, sensors and mechanical components have appeared on the market. This makes the design of small intelligent autonomous robots possible [1], [2], [3] and allows gaining knowledge in building such micro systems [4], [5]. In order to make the recent results accessible to a large field of applications, a modular concept with 'plug and play' modules is of high importance. Key points of these modules and of the whole micro robot are robustness, simplicity and low cost. Particularly low cost makes the system also accessible to the toy market and not only for research. Using the same product in different sectors and applications brings further improvements in technology and costs.

One of the motors for our development was also to build a micro robot that can be used by peoples without special capabilities in engineering, like researchers in artificial life, biologist [7] and maybe also children. Thus the system should be extendable dependent on its use. Various modules have therefore been developed or are under development: distance sensors, short and long distance communication abilities, vision and grippers. Various demonstrations have been established or will be established to show the capabilities on real examples and to trigger new applications. In the next chapters we will show how we managed to build a complete and modular micro robot still keeping it simple and inexpensive.

\section{Hardware design}

\subsection{Components}

The principal components of a autonomous mobile micro robot are actuators, microcontroller, batteries, sensors and the basic electronics.

As actuators (motors responsible of the displacement) we have chosen watch type motors (LAVET [8]). They have a very low power consumption $(0.4 \mathrm{~mA})$, they are highly optimized (several years in the swiss watch industry), easy to control (directly driven from the microcontroller pins) and the gear is included in the motor block.

The system is controlled by a PIC16F84 microcontroller from Microchip [9], which is also characterized by a low power consumption (about $0.6 \mathrm{~mA} @ 4 \mathrm{MHz}$ ). Other important features are its size (SOIC18) and the integrated 
one Kwords of flash program memory. This allows the end user of the robot to change the control program within minutes according to its special needs, particular application or new extension hardware.

The batteries ( 2 button cells from Varta V377) are also thought to be used in watches. They fit perfectly to the robot dimensions. The problem with this kind of batteries is the low maximal current due to the high internal resistance. We use accumulators on some prototypes, but they have a much lower capacitance/volume ratio.

\subsection{Base module}

Besides the sensors, which strongly depend on the particular application, all the other principal components are integrated in the base module. That are: the microcontroller, the resonator, two batteries, the power switch, two watch motors with an aluminum wheel and a rubber tire, the extension bus (connector), one capacitor and one resistor. This main module is built as compact and robust as possible with a main emphasis on low cost production.

The chosen watch motors have 4 electrical contacts that can directly be soldered onto a circuit board. They are mounted together with the other electronic components on a flexible circuit board and bent in the required vertical position during the assemble process. The other electric components remain in the horizontal plane. The flexible print is mounted within a plastic frame (figure 2) and the motors are rigidly attached to it with two screws on each side. This plastic frame holds also the two batteries and the extension modules that can be fixed on top of the basic module.

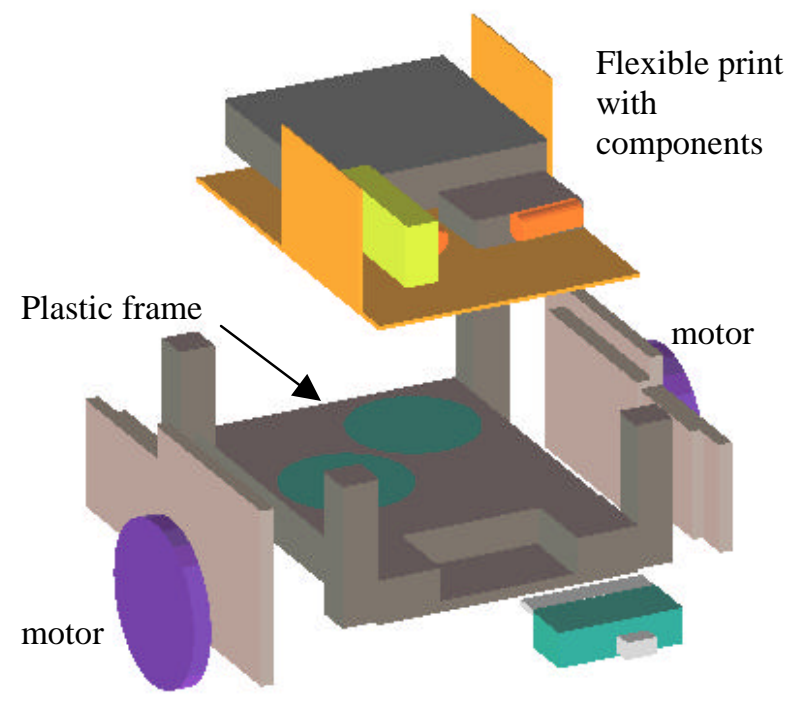

Figure 2. Assembling the base module

The design of the plastic frame is optimized for low cost production. It can either be manufactured by a milling machine all from one side (top) or produced by plastic injection. Another advantage of this kind of plastic structure is that it allows the wheels to move up so that they are protected against mechanical shocks. The other parts that deform in case of shock, are the rubber tires. The robot is thus protected from improper manipulations.

\subsection{Modularity}

The system and its functionality can be extended by just plugging on new modules on top of the base of the robot. The base module has a bus connector with digital in- and outputs, power supply and a serial port.
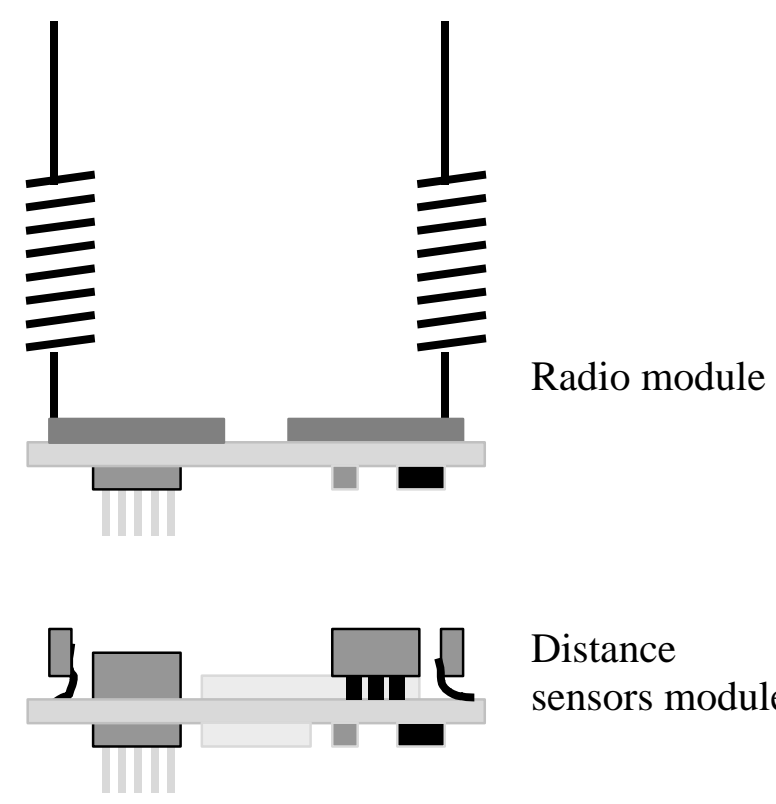

Distance sensors module

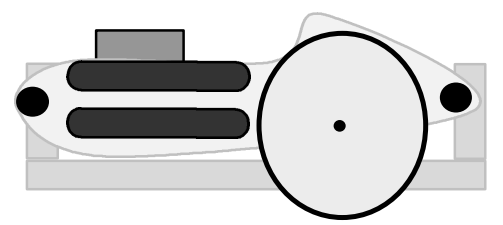

Base module

The modules developed and successfully used until now are: The distance sensors module, the radio communication module, the infrared communication module, the Maze module and the programming-debugging module.

\subsection{Distance sensors module}

This extension is equipped with 4 proximity sensors, an AD converter to read the analogue sensors and a multiplexer to select the different channels. The sensors are SFH900 having an infrared LED and a phototransistor in one package. This allows to detect obstacles and particular situations but also to communicate locally between 2 robots. Obstacles are seen up 
to a distance of $2 \mathrm{~cm}$ and the infrared communication allows local communication between robots up to $4 \mathrm{~cm}$.

\subsection{Radio module}

This module contains the HX1000 device as transmitter and the RX1020 device as receiver, both working at 433.92 $\mathrm{MHz}$. With this transceiver the robot is able to communicate via a radio link with other robot or with a host computer. This feature is of high interest to exchange information between robots even if they don't see each other or to supervise the status of a robot by means of a host computer. The transmission of data in both directions was tested up to a distance of about 10 meters.

\subsection{Infrared communication module}

When only unidirectional communication to the robot is necessary, a cheap infrared receiver module with low power consumption $(0.2 \mathrm{~mA})$ can be used. The components of this unit are a photodiode, an amplifier and a filter. With a simple infrared remote control it is then possible to guide the micro robot and to preprogram certain tasks.

\section{Control strategy}

The on board computational power of a micro robot will always be limited due to limited energy and space. In our case, where computation power is very limited ( 8 bit microcontroller@4 MHz),we adopted a two-stage control strategy.

\subsection{Local control}

The lower control stage is implemented on the robot and controls the motors, the sensors and the external communication. The robot is thus autonomously able to run avoiding obstacles, follow walls, detect corridor crossings and see another robot. It is very important that at least these functions are solved autonomously on the robot itself in order to avoid saturation of the communication channel with commands from external agents.

Typically, the external agent could command the robot to go straight. After decoding the command, the robot drives the motors to move and, reading the distance sensors, it avoids obstacles without any external intervention. Another typical command could be 'follow the right wall'. The robot decodes this command, it looks for a wall on the right side, follows this wall keeping always the same distance and automatically stops when it finds the end of that wall.

\subsection{Global control}

At a higher level of the control system, there are intelligent external units (PC, human operator) that have the capability to perform high level decisions. This units command the robot and get back some kind of feedback information in order to close the control loop. An example is a human with an infrared remote control able to send the commands forward, backward, right, left, follow right wall, follow left wall and finally stop. In this case the feedback is visual and is performed by the operator and his eyes.

In another experimental set-up with our micro robots we connected it to the Internet. The human operator can operate the micro robot through the Internet using some additional path planing and visual feedback support running on the server PC. This allows the user to guide the robot through a maze by only selecting the goal and some via-points. The sequence of the control commands are then generated using the vision signal of a camera installed above the robots environment.

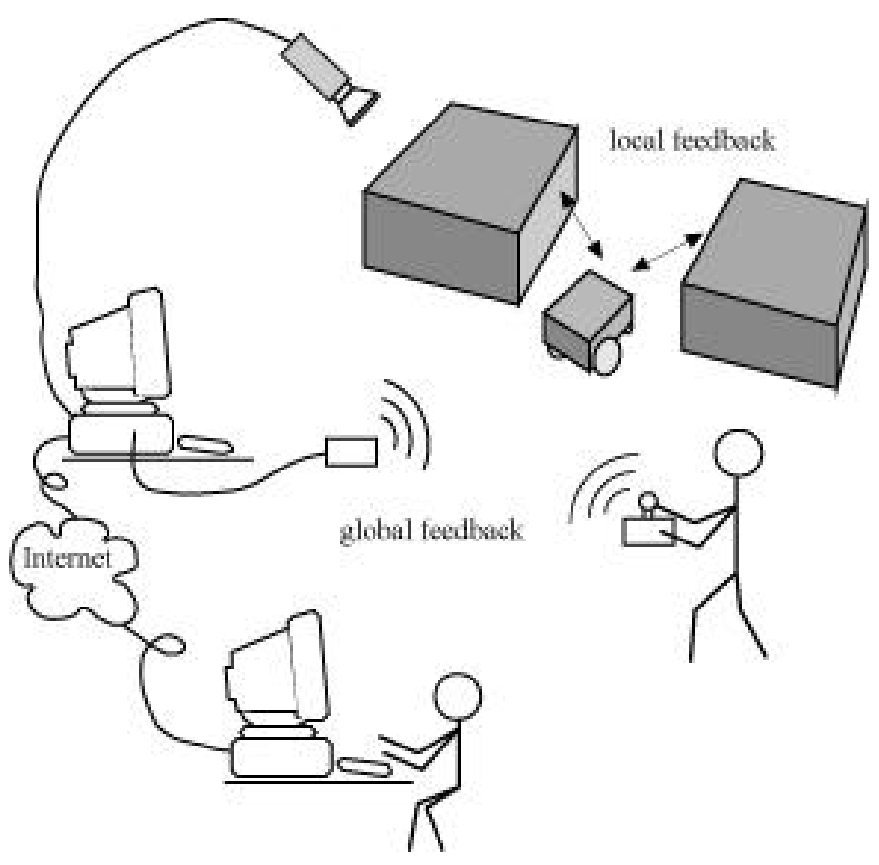

Figure 4. Two stage control strategy

\section{Performance demonstrators}

\subsection{Maze Contest}

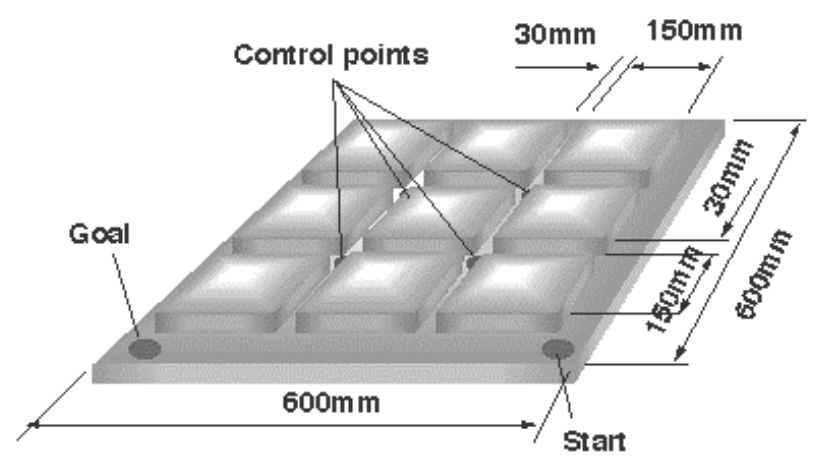

Figure 5. Maze Contest ground 
At the origin of this project some years ago, the idea was to build a micro robot to participate at the "International Micro Maze Contest" in Nagoya, Japan. In fact, an old prototype of the same family of robots, took part in the 1996 Micro Maze Contest and won the second prize. The new robot Alice, with the distance module and the infrared communication module, is able to fulfill the task of the category 2 of this contest (Wireless Autonomous Mobile Robots). Our approach for the contest is the following: the control path is taught to the robot by giving him a sequence of basic commands (first left, than straight, next right ...). After this teaching phase, which can be done with a very simple handhold IR-interface, the robot is able to fulfill the task autonomously.

\subsection{Internet Control}

A very nice demonstrator where Alice is used, is a set-up of $1 \mathrm{~m} \times 1 \mathrm{~m}$ that will be soon available on the Internet. Five Alices with distance and communication modules are located in this set-up. Each single robot can be remote controlled by a different user through Internet. Besides driving his robot, the users can also communicate using a chat-line with the users of the other robots. Three users, could for example, join their efforts to displace a big and heavy object which is too heavy for just one robot.

Figure 6. Internet demonstrator ground

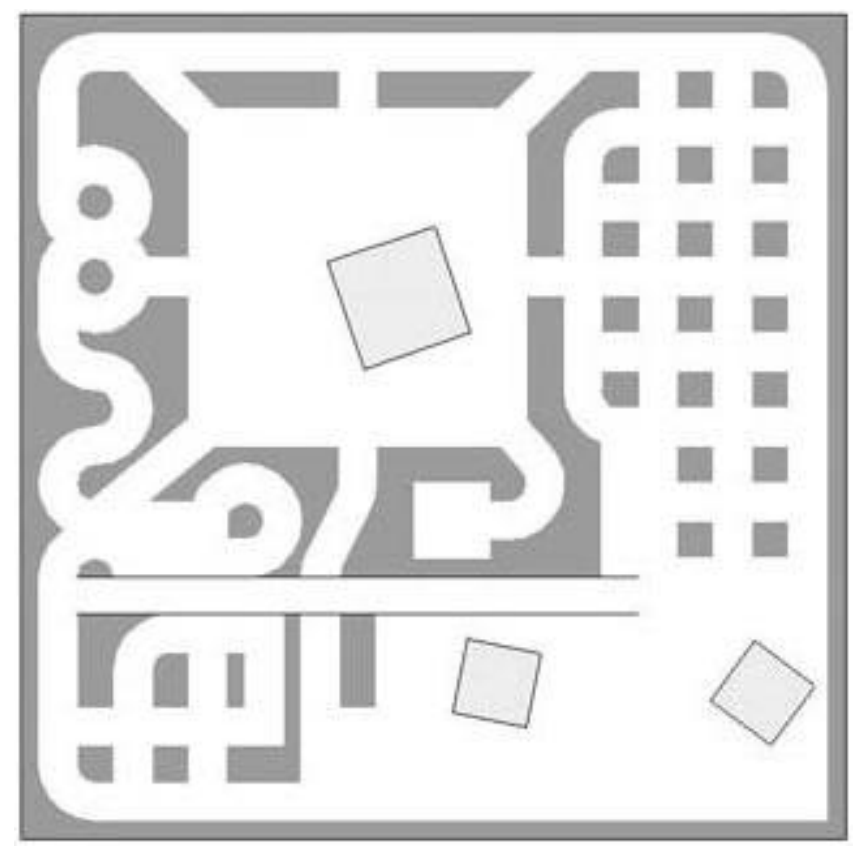

\subsection{Solve the Labyrinth}

The goal of this demonstrator is to show the potential of the radio communication for multi-robot cooperation. They help each other to find the way out of the labyrinth. The different stages of the structure is symbolically explained beneath the labyrinth set-up (see below):

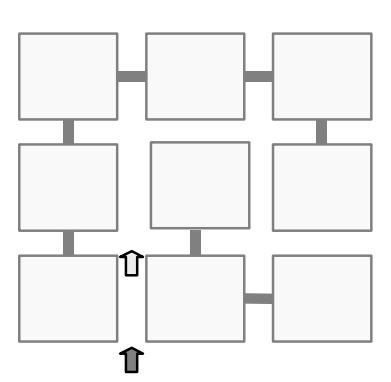

$\Rightarrow$ Robot 1 (master): runs straight to $\Rightarrow$ the next intersection, then tells the Robot 2 to follow.

Next: turns to the left.

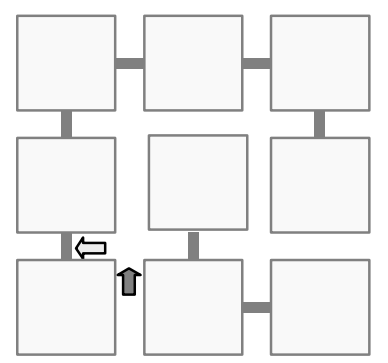

$\Rightarrow$ Robot 1 (master): hits the wall, makes a U-turn and sends to Robot 2 to go ahead.

Next: becomes slave and goes back to intersection.

$\Rightarrow$ Robot 2 (slave): runs to the intersection and waits for instructions from Robot 1.

Next: becomes master and goes straight on.

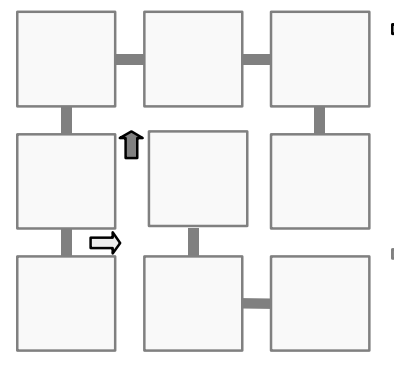

$\Rightarrow$ Robot 1 (slave): waits at the intersection for a command from Robot 2.

Next: turns left and follows Robot 2 .

$\Rightarrow$ Robot 2 (master): at the intersection, it calls the Robot 1 to follow.

Next: turns left.

\section{Conclusion}

In this paper, the micro mobile robot Alice and its features has been presented. Its principal characteristics, being also the main advantages, are the followings:

- It is possible to produce it at very low costs thanks to the simple design and to the low cost of the components.

- It has an extremely low power consumption that allows exceptionally long power autonomy (until 10 hours of work).

- It has a modular concept both in software and in hardware. This allows flexibility for the developing and for new applications.

- It exists a set of extension modules, examples of software and some demonstrators. This is of a great help for future developments and users.

The technical data of the autonomous micro robot Alice are specified in the following table. 


\begin{tabular}{|l|l|}
\hline Dimensions of the base & $21 \times 21 \times 12 \mathrm{~mm}$ \\
\hline + distance sensors module & $21 \times 21 \times 14 \mathrm{~mm}$ \\
\hline + IR communication module & $21 \times 21 \times 18 \mathrm{~mm}$ \\
\hline Velocity & $20 \mathrm{~mm} / \mathrm{s}$ \\
\hline Power consumption & $4 \mathrm{~mW}-7 \mathrm{~mW}$ \\
\hline System autonomy & up to 10 hours \\
\hline Infrared remote communication & $6 \mathrm{~m}, 500 \mathrm{bps}$ \\
\hline Infrared local communication & $4 \mathrm{~cm}, 500 \mathrm{bps}$ \\
\hline Radio communication & $10 \mathrm{~m}, 1000 \mathrm{bps}$ \\
\hline
\end{tabular}

Table 1: Specifications of the autonomous micro robot Alice

\section{References}

[1] H. Ishihara, T. Fukuda. Miniaturized Autonomous Robot. SPIE, vol.3202, pp. 191-6, 1998

[2] J. McLurkin. Using Cooperative Robots for Explosive Ordonance Disposal, MIT AILab, 1996

[3] LAMI-EPFL, Switzerland. The Microrobots Jemmy and Inchy. http://diwww.epfl.ch/lami/mirobots/1 cubes.html

[4] R. Büchi; D. Rohrer; C. Schmid; R. Siegwart. A fully Autonomous Mobile Mini Robot. SPIE Photonics EAST '95, Philadelphia, pp. 50-3, 1995

[5] R. Büchi, G. Caprari, V. Vuskovic, R. Siegwart. A remote controlled mobile mini robot. Proc. of the $7^{\text {th }}$ International Symposium on Micro Machine and Human Science, pp. 203-6, 1996

[6] J.D. Nicoud. Microengineering: when is small too small? Nanoengineering: when is large too large? $6^{\text {th }}$ Symposium on Micro Machine and Human Science, pp. 1-6, 1995

[7] F. Mondada, E. Franzi, P. Ienne. Mobile robot miniaturization: A tool for investigation in control algorithms. Proc. of the $3^{\text {rd }}$ International Sym. On Experimental Robotics, pp. 501-513, 1993

[8] Bernety, J. -C.: Mype LAVET, Bulletin Annuel de la SSC. Vol. X - 1981

[9] Microchip Technology Inc., 2355 West Chandler Blvd. Chandler, AZ 85224-6199 